\title{
La historia del COVID-19 no terminará con el control del SARS-CoV-2. ¿Qué se debe esperar de las comunidades rurales de América Latina más allá de la pandemia. Una perspectiva neurológica The COVID-19 history will not end with the control of SARS-CoV-2. What should be expected in rural communities of Latin America beyond the pandemic? A neurological perspective
}

Oscar H. Del Brutto

La pandemia de Enfermedad por Coronavirus 2019 (COVID-19) causada por el Síndrome Respiratorio Agudo Severo por Coronavirus-2 (SARS-CoV-2) ha afectado a más de 150 millones de personas hasta finales de abril de 2021, y se espera que afecte a muchos más durante el próximo año. Con una tasa de mortalidad de alrededor del $2 \%$ en las personas infectadas, millones de sobrevivientes de COVID-19 quedarán con secuelas crónicas de esta enfermedad. El término COVID prolongado (LongCOVID) se ha acuñado para definir la persistencia de manifestaciones clínicas o anomalías de laboratorio que persisten más allá de la fase aguda de la enfermedad (28 días)., ${ }^{1,2}$ Una revisión sistemática y meta análisis reciente de estudios publicados hasta diciembre de 2020, identificó 55 efectos a largo plazo del COVID-19, muchos de ellos manifestados por compromiso del sistema nervioso central y periférico? Según ésta y otras revisiones, las secuelas neurológicas más importantes incluyen deterioro cognitivo, trastornos del sueño, dolor de cabeza crónico, secuelas de accidente cerebrovascular (el accidente cerebrovascular a menudo ocurre durante la fase aguda de la enfermedad), sarcopenia, fatiga crónica, ageusia y anosmia, pérdida de audición y secuelas neuropáticas de anomalías agudas de los nervios periféricos (incluido el síndrome de Guillain-Barré) $)^{2-4}$ Algunas de estas secuelas neurológicas a largo plazo ocurren en pacientes que solo experimentaron COVID-19 leve y pueden persistir varios meses después del episodio agudo., ${ }^{5,6} \mathrm{La}$ evidencia actual, sin embargo, no permite concluir si estos déficits neurológicos se solucionarán o mejorarán con el tiempo.

Varios comentarios editoriales, publicados durante los primeros meses de la pandemia, anticiparon las devastadoras consecuencias que tendría el SARS-CoV-2 en los entornos rurales de los países en desarrollo ${ }^{7-9}$ La pobreza, junto con el analfabetismo y las desigualdades sanitarias, fueron señalados, entre otros factores, como posibles responsables de este escenario sombrío. Más de un año después del inicio de la pandemia, la información es aún bastante limitada sobre la situación de las poblaciones rurales en el contexto de esta pandemia. Sin embargo, los escasos datos disponibles sugieren que una vez que el virus se introduce en una población rural determinada, se propaga rápidamente, causando una importante mortalidad inicial e infectando a una proporción considerable de la población $^{10-12}$ Además, datos recientes provenientes de una población rural severamente afectada por la pandemia de SARS-CoV-2 proporcionaron pruebas contundentes de las secuelas neurológicas tardías mencionadas anteriormente, al mostrar una frecuencia significativamente mayor de deterioro cognitivo seis meses después de una infección leve sintomática de SARS-CoV-2 en comparación con individuos no infectados, 5 y un deterioro significativo en la calidad del sueño entre los individuos seropositivos en comparación con sus contrapartes seronegativas. ${ }^{6}$ La singularidad de esos estudios residió en que los participantes habían sido evaluados antes y después
School of Medicine, Universidad Espíritu Santo - Ecuador, Samborondón, Ecuador
Correspondencia:

Oscar H. Del Brutto, MD.

Urbanización Toscana, Apt 3H, Km 4.5 vía Puntilla-Samborondón, 092301,

Samborondón-Ecuador.

E-mail: oscardelbrutto@hotmail.com 
del brote de SARS-CoV-2 mediante las mismas pruebas diagnósticas y con el mismo personal sanitario.

Las manifestaciones neurológicas asociadas con la infección aguda por SARS-CoV-2 ocurren con mayor frecuencia durante la segunda semana del inicio de la enfermedad y se observan especialmente en pacientes críticos. Por el contrario, las secuelas neurológicas tardías tienden a manifestarse varios meses después de la infección inicial y no necesariamente ocurren en pacientes con una enfermedad complicada que requiera hospitalización o el uso de oxigenoterapia. La patogenia de las complicaciones neurológicas agudas del COVID-19 ha sido debatida. Sin embargo, algunos estudios ayudaron a proporcionar pistas para una mejor comprensión de la aparición de tales complicaciones. Los accidentes cerebrovasculares se han asociado con enfermedad oclusiva de grandes vasos, embolia cerebral cardiogénica y vasculitis. ${ }^{13}$ Las encefalitis y encefalopatías ocurren como resultado del síndrome de liberación de citokinas ${ }^{14} \mathrm{o}$ debido a la invasión directa del sistema nervioso central por el SARS-CoV-2, ya que el receptor utilizado por el virus para entrar a las células la enzima convertidora de angiotensina-2 - se expresa en neuronas y células gliales. ${ }^{15}$ Las neuropatías ocurren debido a daño de los nervios periféricos o craneales secundario a una respuesta inflamatoria post-infecciosa observada poco después de la fase aguda de la enfermedad. ${ }^{16}$

Por otro lado, es poco lo que se conoce acerca de los mecanismos subyacentes a las secuelas neurológicas tardías de la infección por SARS-CoV-2. Se ha postulado que el SARS-CoV-2 por sí mismo puede ser la causa de este tipo de secuelas en pacientes con COVID-19. ${ }^{17}$ La puerta de entrada del SARS-CoV-2 al sistema nervioso central es con mayor frecuencia el epitelio nasal que contiene fibras del nervio olfatorio. Luego, el virus viaja al bulbo olfatorio (a través de la lámina cribiforme) y se disemina por transferencia trans-sináptica a las estructuras límbicas $\mathrm{y}$, posteriormente, a las partes más profundas del cerebro (incluidos los ganglios basales y el tronco encefálico). Esto se ha sugerido luego de estudios de TC con tomografía por emisión de positrones, que muestran una captación anormal de flúor-18 fluorodesoxiglucosa (hipometabolismo) en las estructuras límbicas, la corteza frontal y orbito-frontal, la circunvolución del cíngulo y el tálamo e hipotálamo. ${ }^{18}$ Una hipótesis alternativa sugiere que hay anomalías en el metabolismo cerebral, las que pueden estar relacionado con mecanismos inflamatorios o autoinmunes. ${ }^{19}$ Esta hipótesis se encuentra respaldada por el hallazgo de biomarcadores inflamatorios en LCR de pacientes con cáncer con encefalopatía relacionada con COVID-19, la cual se desarrolla tres semanas después del inicio de la enfermedad junto con la ausencia de niveles detectables de SARS-CoV-2 por RT-PCR en el LCR ${ }^{20} \mathrm{En}$ los supervivientes de COVID-19 que presentan secuelas neurológicas tardías, también es posible que una respuesta inflamatoria inicial más paucisintomática pueda manifestarse varios meses después de la infección aguda, lo cual, a su vez, conduce a una respuesta inmunológica exagerada. ${ }^{21}$

En entornos rurales remotos, es fundamental cuantificar las secuelas neurológicas tardías asociadas con el COVID-19 para poder evaluar su impacto en los ya limitados sistemas de salud. En todo el mundo, los neurólogos no están disponibles en estas comunidades y es posible que los médicos generales no puedan manejar completamente el diagnóstico del tratamiento de estas secuelas. Incluso en sociedades altamente desarrolladas, la escasez de neurólogos en entornos rurales es evidente. ${ }^{22}$

La telemedicina ha surgido como una alternativa ideal para compensar la falta de neurólogos (y otros especialistas) en entornos remotos. La telemedicina reduce los riesgos y costos de transporte de pacientes y permite la interacción en tiempo real entre médicos y pacientes altamente especializados. Sin embargo, un problema importante con este avance tecnológico en las áreas rurales de los países en desarrollo es la mala conectividad, algo que va a ser muy difícil de superar, por lo menos en los próximos años. ${ }^{23}$

Más recientemente, la vacunación masiva ha surgido como la opción más factible para el control de la pandemia. ${ }^{24}$ Sin embargo, varios problemas logísticos y económicos harán que las personas que viven en las zonas rurales de los países en desarrollo sean las menos favorecidas con esta intervención y probablemente se necesitarán años para completar esta tarea. Mientras tanto, muchas más personas contraerán la enfermedad saturando así los limitados recursos sanitarios en estos poblados y dejando aún menos recursos para el manejo de pacientes con complicaciones neurológicas tardías del COVID-19. Si bien el panorama no parece alentador, esperemos que los gobiernos regionales tomen medidas para mejorar la conectividad en zonas rurales y, de esa manera, sea posible valorar telemáticamente a estos pacientes.

\section{Referencias}

1. Mandal S, Barnett J, Brill SE, et al. "Long-COVID": a cross-sectional study of persisting symptoms, biomarker and imaging abnormalities following hospitalization for COVID-19. Thorax. http://doi. org/10.1136/thoraxjnl-2020-215818.

2. Sudre CH, Murray B, Varsavsky T, et al. Attributes and predictors of long COVID. Nat Med http://doi. org/10.1038/s41591-021-01292-y.

3. Lopez-Leon S, Wegman-Ostrosky T, Perelman C, et al. More than 50 long-term effects of COVID-19: a systematic review and meta-analysis. medRxiv. http://doi.org/10.1101/2021.01.27.21250617.

4. Wang F, Kream RM, Stefano GB. Long-term respiratory and neurological sequelae of COVID19. Med Sci Monit. 2020; 26: e928996. http://doi. org/10.12659/MSM.928996. 
5. Del Brutto OH, Wu S, Mera RM, Costa AF, Recalde BY, Issa NP. Cognitive decline among individuals with history of mild symptomatic SARS-CoV-2 infection. A longitudinal prospective study nested to a population cohort. Eur J Neurol. https://doi. org/10.1111/ene.14775.

6. Del Brutto OH, Mera RM, Costa AF, Recalde BY, Castillo PR. Sleep quality deterioration in middleaged and older adults living in a rural Ecuadorian village severely struck by the SARS-CoV-2 pandemic. A population-based longitudinal prospective study. Sleep. https://doi.org/10.1093/sleep/zsab041.

7. Miller MJ, Loaiza JR, Takyar A, Gilman RH. COVID-19 in Latin America: novel transmission dynamics for a global pandemic? PloS Negl Trop Dis. 2020; 14: e0008265. https://doi.org/10.1371/ journal.pntd.0008265.

8. Meneses-Navarro S, Freyermuth-Enciso MG, Pelcastre-Villafuerte BE, Campos-Navarro R, Meléndez-Navarro DM, Gómez-Flores-Ramos L. The challenges facing indigenous communities in Latin America as they confront the COVID-19 pandemic. Int J Equity Health. 2020; 19: 63. https://doi. org/10.1186/s12939-020-01178-4.

9. Burki T. COVID-19 in Latin America. Lancet Infect Dis. 2020； 20: 547-548. https://doi.org/10.1016/ S1473-3099(20)30303-0.

10. Del Brutto OH, Costa AF, Mera RM, Recalde BY, Bustos JA, García HH. SARS-CoV-2-related mortality in a rural Latin American population. Int J Infect Dis. 2020; 99: 226-228. https://doi.org/10.1016/j. ijid.2020.08.003.

11. Del Brutto OH, Costa AF, Mera RM, Recalde BY, Bustos JA, García HH. SARS-CoV-2 in rural Latin America. A population-based study in coastal Ecuador. Clin Infect Dis. 2020; https://doi. org/10.1093/cid/ciaa1055.

12. Del Brutto OH, Costa AF, Mera RM, Recalde BY, Bustos JA, García HH. Late incidence of SARSCoV-2 infection in a highly endemic remote rural setting. A prospective population-based cohort study. Pathog Glob Health. 2020; 114: 457-462. https://doi.org/10.1080/20477724.2020.1826152.

13. Spence JD, de Freitas GR, Pettigrew LC, et al. Mechanisms of stroke in COVID-19. Cerebrovasc Dis 2020; 49: 451-458. https://doi.org/10.1159/000509581.

14. Perrin P, Collongues N, Baloglu S, et al. Cytokine release syndrome-associated encephalopathy in patients with COVID-19. Eur J Neurol 2020; https:// doi.org/10.1111/ene.14491.
15. Natoli S, Oliveira V, Calabresi P, et al. Does SARSCoV-2 invade the brain? Translational lessons from animal models. Eur J Neurol 2020; https://doi. org/10.1111/ene.14277.

16. Abu-Rumeileh S, Abdelhak A, Foschi M, et al. Guillain-Barré syndrome spectrum associated with COVID-19: an up-to-date systematic review of 73 cases. J Neurol 2020; https://doi.org/10.1007/s00415020-10124-x

17. Serrano-Castro PJ, Estivill-Torrús G, CabezudoGarcía P, et al. Influencia de la infección SARS$\mathrm{CoV}-2$ sobre enfermedades neurodegenerativas y neuropsiquiátricas: ¿una pandemia demorada? Neurología. 2020; 35: 245-251. https://doi.org/10.1016/j. nrl.2020.04.002.

18. Guedj E, Million M, Dudouet P, et al. 18F-FDG brain PET hypometabolism in post-SARS-CoV-2 infection: substrate for persistent/delayed disorders? Eur J Nucl Med Mol Imaging. https://doi.org/10.1007/ s00259-020-04973-x.

19. Delorme C, Paccoud O, Kas A, et al. COVID19-related encephalopathy: a case series with brain FDG-PET/CT findings. Eur J Neurol. https://doi. org/10.1111/ene.14478.

20. Remsik J, Wilcox JA, Babady NE, et al. Inflammatory leptomeningeal cytokines mediate delayed COVID-19 encephalopathy. medRxiv. https://doi. org/10.1101/2020.09.15.20195511.

21. Koralnik IJ, Tyler KL. COVID-19: a global threat to the nervous system. Ann Neurol. 2020; 88: 1-11. https://doi.org/10.1002/ana.25807.

22. Neurologists Much Tougher to Find in Rural America - Healthfortnight.com

23. Smith AC, Bensink M, Armfield N, et al. Telemedicine and rural health care applications. J postgraduate Med 2005; 51: 286-293. PMID: 16388171.

24. Pandey SC, Pande V, Sati D, et al. Vaccination strategies to combat novel corona virus SARS-CoV-2. Life Sci 2020; 256: 117956. https://doi.org/10.1016/j. lfs.2020.117956.

Palabras clave: Enfermedad por coronavirus 2019, COVID19, SARS-CoV-2, COVID prolongado, Secuelas neurológicas, comunidades rurales.

Keywords: Coronavirus disease 2019, COVID-19, SARS-CoV-2, Long COVID, Neurological sequelae, rural communities.

Institución Auspiciante: Universidad Espiritu Santo-Ecuador. 